\title{
A Negative Inotropic Effect of Omega-Conotoxin GVIA in Isolated, Blood-Perfused Dog Right Atria
}

\author{
Lei-Ming Ren, Yasuyuki Furukawa, Yasuyuki Karasawa, Makoto Murakami, \\ Shin Takayama, Yasurou Inoue, Shoji Sawaki and Shigetoshi Chiba* \\ Department of Pharmacology, Shinshu University School of Medicine, Matsumoto 390, Japan
}

Received August 19, 1991 Accepted October 28, 1991

\begin{abstract}
Using an isolated, blood-perfused canine atrial preparation, we investigated the effects of omega-conotoxin GVIA (omega-CgTX), a blocker of N-type voltage-operated calcium channels, on the atrial contractile force and sinus rate. Omega-CgTX in a dose range of $0.3-3 \mathrm{nmol}$ gradually attenuated the atrial contractile force in a dose-dependent manner, but did not affect the sinus rate, indicating that it can depress contractility but not pacemaker activity.
\end{abstract}

On the basis of electrophysiological and pharmacological differences, voltage-operated calcium channels (VOCCs) have been subdivided into $\mathrm{L}$-, $\mathrm{N}$ - and T-type VOCCs (1). Ntype VOCCs exist only in neuronal preparations (1-3). Omega-conotoxin GVIA (omega Conus geographus toxin, omega-CgTX), a 27amino acid peptide purified from the fishhunting snail Conus geographus (4), blocks neuronal N- and L-type VOCCs, but not nonneuronal L-type VOCCs in patch-clamp electrophysiological studies $(2,3)$. The stimulation-induced release of neuronal transmitters (noradrenaline, acetylcholine or non-adrenergic, non-cholinergic transmitters) is attenuated by omega-CgTX effectively in various tissues of the rat, rabbit and mouse (5). However, the responses to exogenous administration of noradrenaline, acetylcholine and adenosine triphosphate are not affected by omega-CgTX in the same preparations (5). In 1988, Ichida et al. (6) reported that a synthetic omegaCgTX itself caused a dose-dependent contraction of segments of the stomach fundus and uterus. They suggested that omega-CgTX

*To whom correspondence should be addressed. might have a calcium agonist-like effect on smooth muscles. However, there is yet no information about the cardiac effects of omegaCgTX. Therefore, we evaluated the effects of omega-CgTX on the atrial contractile force and sinus rate in the isolated, blood-perfused canine atrium.

Ten mongrel dogs, weighing from 10 to 24 $\mathrm{kg}$, were anesthetized with sodium pentobarbital $(30 \mathrm{mg} / \mathrm{kg}, \mathrm{i.v})$, and then ventilated artificially with room air. Sodium heparin (500 USP units/kg, i.v.) was administered at the beginning of the perfusion of the isolated atrial preparation, and $200 \mathrm{USP}$ units/kg were given each hour thereafter. Isolated right atrial preparations were obtained from another 10 mongrel dogs. Each dog, weighing from 8 to $13 \mathrm{~kg}$, was anesthetized with sodium pentobarbital $(30 \mathrm{mg} / \mathrm{kg}$, i.v.). After sodium heparin (200 USP units/kg, i.v.) was given, the right atria, wet weight of $11.3 \pm 2.2$ (mean \pm S.D.) $\mathrm{g}$, were excised and immersed in cold Ringer solution. The sinus node artery was cannulated via the right coronary artery and perfused with blood from the carotid artery of the support dog by the aid of a peristaltic pump (Harvard Apparatus model 1210). The 
ventricular margin of the atrium was anchored to a stainless steel bar and placed in a cupshaped glass container which was kept at a constant temperature of $37^{\circ} \mathrm{C}$. The isometric tension was recorded on a thermo-writing oscillograph (Nihon Kohden) by a forcedisplacement transducer (Nihon Kohden AP620G) connected to the superior part of the atrium with a silk thread. A pair of bipolar silver electrodes was brought into contact with the epicardial surface of the isolated atrium to record the atrial electrogram, from which the atrial rate was derived by a cardiotachometer (Nihon Kohden AT 600G). The details of this preparation have been described previously (7). Omega-conotoxin GVIA (omega-CgTX, Sigma Chemical Co., USA) was dissolved in $0.5 \% \mathrm{~W} / \mathrm{V}$ bovine serum albumin in distilled water. OmegaCgTX was injected into the sinus node artery at a volume of 0.01 to $0.03 \mathrm{ml}$ over a period of $4 \mathrm{sec}$.
Experiments were divided into two groups. In 5 atria, we injected omega-CgTX at doses of $0.3,1$ and $3 \mathrm{nmol}$ into the sinus node artery of each preparation. In another 5 atria, the vehicle was injected at the volume of $0.01,0.3$ and $0.01 \mathrm{ml}$, corresponding to the volumes in the omega-CgTX treatment group. Each dose of omega-CgTX or vehicle was given at $40-\mathrm{min}$ intervals. The atrial contractile force and sinus rate were determined in four periods: before administration of omega-CgTX or vehicle and 30 min after each administration of $0.3,1$ and $3 \mathrm{nmol}$ of omega-CgTX or after each administration of vehicle. Values presented here are means \pm S.E.M. Two-way analysis of variance with randomized block design was used to compare the cardiac effects of omega-CgTX with those of the vehicle. The experimental groups and four periods were considered to be fixed factors. $P$ values less than 0.05 were considered statistically significant.

Figure 1 shows the effects of omega-CgTX

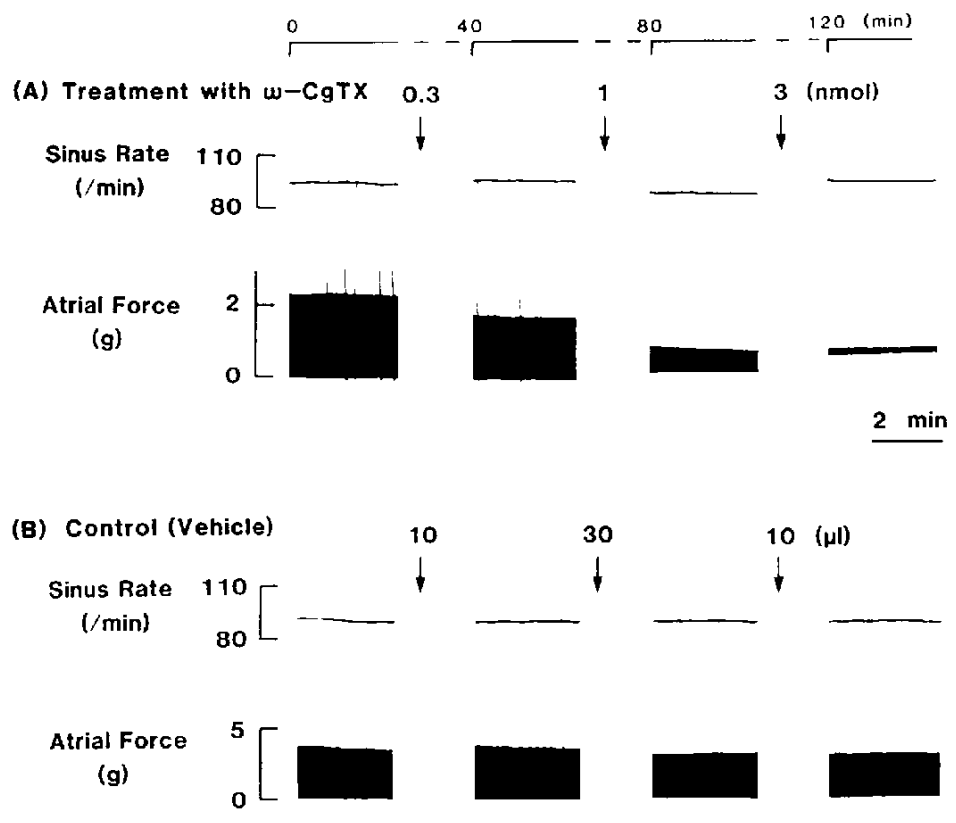

Fig. 1. Effects of omega-CgTX $(0.3,1$ and $3 \mathrm{nmol}, \mathrm{A})$ and vehicle (B) on the sinus rate and atrial contractile force in isolated, blood-perfused dog atria. Recordings represent the effects at 30 min after each administration of omega-CgTX or vehicle, except for the recordings before treatment. 
on the sinus rate and atrial contractile force. Omega-CgTX at doses of $0.3,1$ and $3 \mathrm{nmol}$ did not affect the sinus rate, but it depressed the atrial contractile force in a dose-dependent manner (Fig. 1A). The negative inotropic effect of omega-CgTX developed gradually. In the control group (vehicle, Fig. 1B), the atrial contractile force decreased slightly. However, the sinus rate did not change within $120 \mathrm{~min}$. Summarized data are shown in Fig. 2. OmegaCgTX attenuated the atrial contractile force dose-dependently and the inhibition of the atrial contractile force induced by omega- $\mathrm{CgTX}$ was significantly $(P<0.001)$ greater than that induced by the vehicle. The decreases in the atrial contractile force induced by omega$\mathrm{CgTX}$ at $3 \mathrm{nmol}$ and that by vehicle were $65 \%$ and $23 \%$, respectively. Although omega-CgTX raised the diastolic atrial tension in Fig. 1, the increase in diastolic tension induced by omega-CgTX was not significant in 5 experiments. Neither omega-CgTX nor vehicle changed the sinus rate significantly (Fig. 2).

In the present study, we demonstrated that omega-CgTX, a neural N-type VOCCs block$\mathrm{er}$, attenuated the atrial contractile force in a dose-dependent manner, but did not affect the sinus rate in the isolated, perfused dog atrium.

Patch-clamp studies (8) have demonstrated that omega-CgTX is a selective and potent inhibitor of $\mathrm{L}$ - and $\mathrm{N}$-type calcium channels in dorsal ganglion and sensory, sympathetic and hippocampal neurons of vertebrates but not in cardiac, skeletal or smooth muscle cells. De Luca et al. (5) reported that omega-CgTX inhibited positive chronotropic responses to field stimulation of adrenergic nerves in rat and mouse atria, but did not affect responses to noradrenaline and tyramine. They did not show the effects of omega-CgTX itself on atrial rate and atrial contractile force. N-type VOCCs exist only in neuronal tissue $(1-3)$. However, the atrial preparation is isolated from the regulation of the central nervous system. Thus, it is unlikely that the inhibition by omega-CgTX of the atrial contractile force is attributed to its neuronal $\mathrm{N}$-type VOCCs inhibition in atrial muscle. That is, omega-CgTX
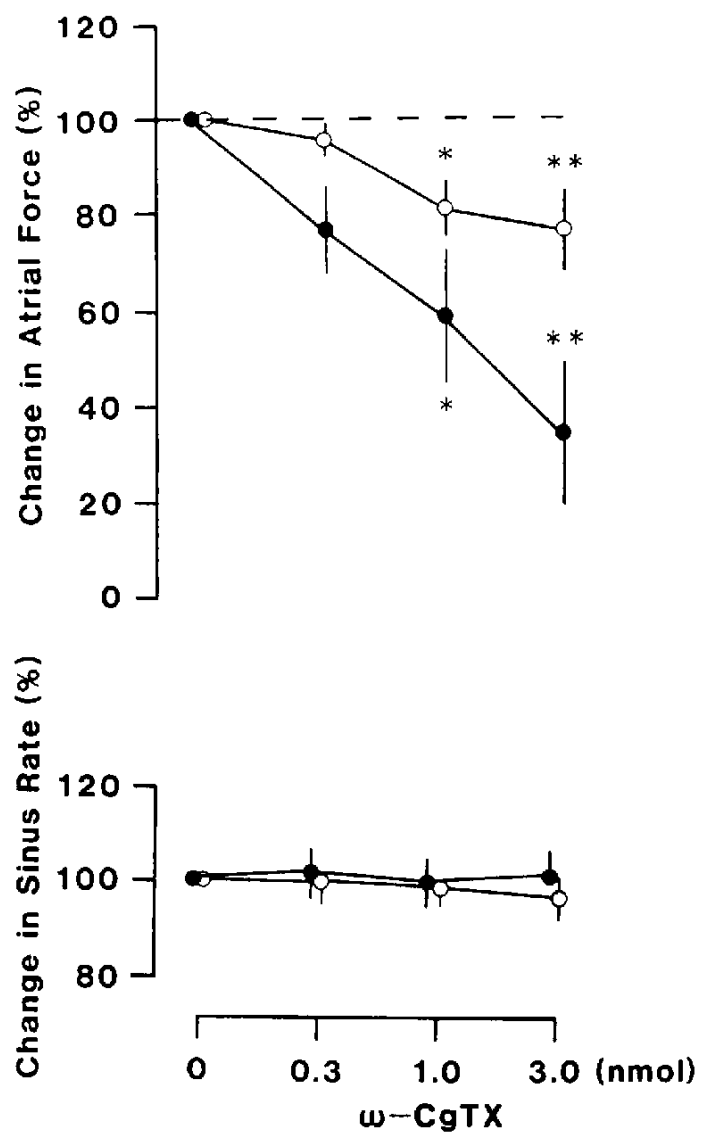

Fig. 2. Percentage changes in atrial contractile force and sinus rate produced by omega-CgTX (closed circles) at doses of $0.3,1$ and $3 \mathrm{nmol}$ and by vehicle (open circles, control) injected into the sinus node artery in isolated, blood-perfused dog atria. Each dose of the drug or vehicle was administered at $40-\mathrm{min}$ intervals. Symbols (open and closed circles) represent means and vertical bars show S.E.M. Asterisks represent statistical significances vs. the pre-drug value analyzed by Bonferroni $t$-test: ${ }^{*} \mathrm{P}<0.05,{ }^{*} \mathrm{P}<0.01$. Basal levels of atrial contractile force and sinus rate for the control group and omega-CgTX group were 4.3 \pm 0.7 (mean \pm S.E.M.) g and $97 \pm 4$ beats $/ \mathrm{min}(\mathrm{n}=$ 5 ) and $3.0 \pm 0.2 \mathrm{~g}$ and $100 \pm 4$ beats $/ \mathrm{min}(\mathrm{n}=5)$, respectively.

may depress the atrial contractile force directly.

It was reported that omega-CgTX caused dose-dependent contraction in segments of the rat stomach fundus and uterus (6). These contractile responses to omega- $\mathrm{CgTX}$ depended 
upon the presence and/or the influx of extracellular calcium and these responses were inhibited by calcium antagonists, suggesting that omega-CgTX might have a calcium agonist-like effect on smooth muscles (6). However, omega-CgTX caused only a negative inotropic response in the isolated, bloodperfused dog atrium. An L-type calcium channel agonist, Bay $\mathrm{k} 8644$, induces positive inotropic and chronotropic cardiac effects in this preparation, and L-type calcium channel antagonists induce negative cardiac effects (9). Thus, the L-type calcium channel antagonistic action may not be a main mechanism for decreasing the atrial contractility without pacemaker activity. Although omega-CgTX has no direct pressor action in anesthetized rats (10), we need further studies, including an assessment of the vascular effects of omega-CgTX, to clarify the precise mechanism of the negative inotropic effect of this compound.

\section{REFERENCES}

1 Nowycky, M.C., Fox, A.P. and Tsien, R.W.: Three types of neuronal calcium channel with different calcium agonist sensitivity. Nature 316, 440-443 (1985)

2 McCleskey, E.W., Fox, A.P., Feldman, D. and Tsien, R.W.: Different types of calcium channels. J. Exp. Biol. 124, 177-190 (1986)

3 McCleskey, E.W., Fox, A.P., Feldman, D., Olivera, B.M., Tsien, R.W. and Yoshikami, D.: The peptide toxin $\omega$-CgTX blocks particular types of neuronal $\mathrm{Ca}^{++}$channels. Biophys. J. 49, 431a (1986)

4 Kerr, L.M. and Yoshikami, D.: A venom peptide with a novel presynaptic blocking action. Nature 308, 282-284 (1984)

5 De Luca, A., Li, C.G., Rand. M.J., Reid, J.J., Thaina, P. and Wong-Dusting, H.K.: Effects of $\omega$-conotoxin GVIA on autonomic neuroeffector transmission in various tissues. Br. J. Pharmacol. 101, 437- 447 (1990)

6 Ichida, S., Oka, H., Masada, A., Fujisue, T., Hata, T. and Matsuda, N.: Effects of synthetic $\omega$ conotoxin on the contractile responses of segments of rat ileum, stomach fundus and uterus and guinea pig taenia coli. Japan. J. Pharmacol. 48, $395-405$ (1988)

7 Chiba, S., Kimura, T. and Hashimoto K.: Muscarinic suppression of the nicotinic action of acetylcholine on the isolated, blood-perfused atrium of the dog. Naunyn Schmiedebergs Arch. Pharmacol. 289, 315 - 325 (1975)

8 McCleskey, E.W., Fox, A.P., Feldman, D.H., Cruz, L.J., Olivera, B.M., Tsien, R.W. and Yoshikami, D.: $\omega$-Conotoxin: Direct and persistent blockade of specific types of calcium channels in neurons but not muscle. Proc. Natl. Acad. Sci. U.S.A. 84, 4327-4331 (1987)

9 Furukawa, Y., Ogiwara, Y., Akahane, K. and Chiba, S.: Different antagonism of the positive chronotropic and inotropic responses of the isolated, blood-perfused dog atrium to Bay $k 8644$ by nicardipine and verapamil. Eur. J. Pharmacol. 156, 231 - 237 (1988)

10 Pruneau, D. and Angus, J. A.: Apparent vascular to cardiac sympatholytic selectivity of $\omega$-conotoxin GVIA in the pithed rat. Eur. J. Pharmacol. 184, $127-133(1990)$ 